\title{
A novel strategy to protect Sutton's nevi during forced or voluptuary exposition to sun rays
}

\section{Lorenzo Martini}

\author{
Department of Pharmaceutical Biotechnologies, University of Siena, Via A. Moro 2, 53100 Siena, Italy
}

Corresponding author: Lorenzo Martini, M.Sc., E-mail: martinil163@libero.it

\begin{abstract}
Albeit halo nevi are estimated to be present in approximately $1 \%$ of the general population, and they are found to be more prevalent in people with vitiligo, malignant melanoma or Ullrich-Turner's syndrome, it is not necessary evident that their presence in human body is to be associated to vitiligo and/or melanoma, both genetically and pathophisiologically. In this study I want to demonstrate how it can be safe to expose Sutton's nevi (halo nevi) to all the sun rays (even the most dangerous during all the daylight), employing two natural cosmetic items: the former based on natural juleps made of polychrome algae (and it is to be considered the absorber sun filter) and the latter is a physical sun blocking agent, apt to reflect all the UV rays. The former must be spread onto the halo, the latter, after half an hour, onto the nevus itself.
\end{abstract}

Keywords: Halo nevi; Leukoderma acquisitum centrifugum; Suttos’s nevi; Fucoxanthin; Całkosiński-Chrószcz method

\section{INTRODUCTION}

Sutton's nevus, alias halo nevus also known as "Leukoderma acquisitum centrifugum," or "Perinevoid vitiligo," even if manifold Authors retain imperfect this definition (and I will explain this diatribe) is a mole that is surrounded by a depigmented ring or 'halo' [1].

Halo nevi are associated mentally and psychologically (and therefore confused) with vitiligo, since they occur commonly in co-presence with vitiligo (and often together with metastatic melanoma), even if the halo nevus does not present the same histological pattern of vitiligo. Sometimes the pale (hypopigmented) areas will spontaneously regress, and pigment returns, too often the hypopigmented areas especially cause of the effect of sun rays may induce in the individual change in appearance of the nevus itself, pain, itch, and infection, all co-factors that could evoke melanoma as well., by the same way sun rays can induce melanoma in albinos $[2,3]$.
Effectively, two are the basic mechanisms whereby the skin can become white $[4,5]$. The former is recognizable when Melanin is synthesized by melanocytes within melanosomes that are transferred into the surrounding keratinocytes. The keratinocytes transport the melanin and melanosomes from the basal layer of the epidermis to the stratum corneum where they are desquamated into the environment [6]. The latter way is when some disorders (certain odd cells) inhibit or retard the production of melanin formation and the skin develops hypopigmentation [5]. Such disorders include, among many others, oculocutaneous albinism, pityriasis alba, tinea versicolor and halo nevus (depigmentosus). In these disorders, melanocytes are present in normal numbers in the epidermis but produce less than normal amounts of melanin. Typically, the skin exhibits mild to marked hypopigmentation.

The formation of a halo surrounding a nevi is believed to occur when the aforesaid "disorders" which are identified as certain white blood cells called CD8+ T lymphocytes destroy the pigment-producing cells of the

\footnotetext{
How to cite this article: Martini M. A novel strategy to protect Sutton's nevi during forced or voluptuary exposition to sun rays. Our Dermatol Online. 2016;8(3):268-271.

Submission: 15.06.2016; Acceptance: 08.08.2016

DOI: 10.7241 /ourd.20173.77
} 
skin (melanocytes). The cause for the attack is hitherto unknown [3]. It is necessary to assert that the white skin of those with vitiligo does not drive to-melanoma skin cancers although the white skin of albinos can lead to melanoma skin, and it must be which has a similar color as vitiligo, is highly susceptible to skin cancer.

Individuals who present a large amount of halo nevi may be confronted to albinos, as far as proneness to melanoma is concerned, whenever they are exposed to excessive sun rays [6].

Halo nevi are estimated to be present in approximately $1 \%$ of the general population, and are found to be more prevalent in people with vitiligo, malignant melanoma or Ullrich-Turner's syndrome [7]. Signs and symptoms of this rare syndrome that affects young girls vary among these manifestations: short and webbed neck, low-set ears, low hairline at the back of the neck, short stature, and swollen hands and feet. Typically, they are without menstrual periods, do not develop breasts, and are unable to have children. Heart defects, diabetes, and low thyroid hormone occur more frequently. Leukoderma acquisitum centrifugum is a special symptom for a complete diagnosis, especially when halo nevi are symmetric in legs. Most people with TS have normal intelligence. even if in some case, somebody, suffers from troubles with spatial visualization such as that needed for mathematics [8]. Vision and hearing problems occur more often [9].

All races and sexes are equally susceptible to this disease, although a familial tendency has been reported. The average age of onset is in a person's teenage years.

Notwithstanding an avalanche of physicians and dermatologists agree with the benefits evoked by treatment of vitiligo by using UVB rays $[10,11]$, it is compulsory to confirm that UVB (administered with the secret hope that a homogeneous colour of the skin surrounding the Sutton's nevus may convince observers that the disease does not exist) UVB rays are utterly dangerous to combat hypopigmentation of haloes, as it would be as UVB rays were applied directly onto albinos' skin with the consequent risk of insurgence of melanoma as well.

Haloes should receive the adequate dose of UVA rays, in order to assume a pinkish colour, and nevi should never be exposed to sun or artificial UV lamps, howbeit when people affected by halo nevi must to stay at the sun (voluptuously or for sake of job), they have to face the problem covering halos with biomaterials (like blue algae which do not absorb all the damaging UVB rays, although are able to absorb the UVA and only afterwards, when the aforesaid biomaterials have performed their substantivity with regards to hypopigmented skin, thus spreading exclusively onto the nevi certain suspensions of inert powders (oxides like magnesia usta or salts like barium sulphate, zinc stearate or magnesium carbonate or minerals like limestone or aragonite, biomaterials like kieselguhr or rice starch) capable to reflect physically all the $\mathrm{UV}$ rays (A, B and $\mathrm{C})$.

\section{MATERIALS AND METHODS}

We have recruited four bricklayers (A,B,C,D, who have to work for 8 hours under the inclement sun while embellishing an ivory-white façade (and underwent involuntarily to albedo effect that multiplies the injuries of sun radiations onto skin), two women (E,F) (40-50y) who loves to stay at the poolside for hours and hours during the worst hours of the diuturnal sunshine and a girl (G) (19y) suffering from the Ullrich-Turner's syndrome, who was forced to expose herself to sun rays for many hours pro day to treat juvenile acne evoked by her congenital hypergonadism.

Cases A,B,C and D presented halo nevi on their trunk and back.

$\mathrm{E}$ and $\mathrm{F}$ presented halo nevi on legs and thighs.

G only asymmetrically on her legs.

I have prepared two cosmetic items I prayed the volunteers to apply on their skin before exposure to the sun, for eight hours pro day.

The first was a hydrogel made of a solution of locust bean gum and a julep of mashed blue, red and brown algae. Amongst blue algae I have chosen the Chlamydomonas nivalis (Bauer) Wille [12], that can be picked up in mountain lakes easily, and amongst the red I preferred to employ the japanese Kousu Ekisu and amonsgst the brown, the Kassou and the Cytosteira amentacea/caespiotsa/brnachycarpa algae, all retrievable in whichever shop that sell food for fish aquariums and all those algae are admitted in INCI for all types of cosmetic items.

The gélée containing the mashed polychrome algae has to be spread half an hour before to apply the second cosmetic product. 
The second cosmetic item, instead, was a paste made of kieselguhr, magnesia usta, barium sulphate and propylene glycol, apt to reflect every type of sun ray or other physical radiation, since it is well known that onto all halo nevi must however and always applied sunscreen systems during summer to prevent sunburn, as the white skin of a halo nevus will burn particularly easily in the sun because it is missing protective melanin pigment at all, and sunburn in these areas are to be reputed extremely prejudicial for human health, driving too often to melanoma.

We would like to repeat that blue algae (retrievable in the deep of manifold lakes in mountains, so that they are commonly called alpine algae) do absorb, thanks to the presence of phycocyanin, all the radiations comprised between 280 and $315 \mathrm{~nm}$ ), and absorb only $50 \%$ of radiations between 315 and $400 \mathrm{~nm}$, meanwhile the red and brown ones, owing to the presence of the fucoxantin, are capable to absorb all the UVA-rays (315-400nm).

This julep is indicated expressly to protect haloes by means of letting them to absorb only the beneficial UVA rays, in order to grow pinkish with no hazard of sunburn and equivalent occurrence of future melanoma.

The purpose of the paste is to cover and protect the nevus itself.

It is well known that pigments (fucoxanthins and phycocyanin) are substantive to the skin keratin, insofar they adhere to the corneocytes and are able to absorb the UVA, so that after the experimentations the halo grows pinkish, but does not undergo sunburn and melanoma.

For this reason it was suggested to apply before the julep to let the pigments to act as substantive agents with regards to the corneocytes. The paste was to applied only onto the single nevi.

We will see that the nevi will remain completely protected, after the removal of the paste, by gentle rubbing with soft sponge.

The experimentation lasted three days (all the days were largely sunny) and among the myriad of tests apt to evaluate the reached decrease of skin inflammation, I have chosen the Całkosiński- Chrószcz method [13], (University of Wrocklaw), based on the evaluation by Infrared Thermography, even if the original method forecasts the evocation of inflammation by the injection of a solution of carrageneean and by the observation of the increase of inflammation and consequent fever in the next 72 hours (generally the increase of temperature in the inflamed area is 3-4 degrees Celsius).

In our case, the method is based upon the contrary strategy: digital infrared imaging referring to the temperature of the halo of the individual who underwent an exposure of 8 hours to sun rays are scored at the end of the first day, when he did not apply no cosmetics apt to block sun rays and the original temperature index was scored.

After three days (that correspond to 72 hours) of application of the two cosmetic items I ideated onto the haloes, the temperature generally, we see, tends to decrease.

\section{Ethics}

This study was performed on human subjects; thus, all patients were aware of the presence of the study and they were fully informed about the drug and its side-effects.

\section{RESULTS}

In Table 1 it is possible to observe how the temperatures decrease in 72 hours, in comparison to the first score, recorded one week before the very first day of complete exposure to sun rays, using the two cosmetic items We created. The temperatures were measured by IRthermography only in the circumscribed areas of the haloes, where the julep was spread.

Original temperatures have been recorded one week before the treatment, object of my research.

In every case it is possible to notice a decrement of inflammation of the halo of the Sutton's nevus exposed to sun rays of an average of $19.9 \%$.

Table 1: Temperatures recorded according to the Całkosiński-Chrószcz method

\begin{tabular}{lcc}
\hline Case & Original temperature & Temperature after $\mathbf{7 2}$ hours \\
\hline A & 36.8 & 30.5 \\
B & 34.6 & 31.5 \\
C & 33.9 & 30.6 \\
D & 35.4 & 32.2 \\
E & 33.6 & 30.9 \\
F & 34.3 & 31.0 \\
G & 32.7 & 30.8 \\
\hline
\end{tabular}




\section{CONCLUSION}

It is interesting to highlight that in Case $\mathrm{G}$ the percentage of inflammation degree is lower (5.9\%) since too often people suffering from Ullrich-Turner's syndrome are endowed by an equipment of melanocytes major than other normal individuals.

\section{Statement of Human and Animal Rights}

All procedures followed were in accordance with the ethical standards of the responsible committee on human experimentation (institutional and national) and with the Helsinki Declaration of 1975, as revised in 2008.

\section{Statement of Informed Consent}

Informed consent was obtained from all patients for being included in the study.

\section{REFERENCES}

1. William DJ, Timoty G. Andrews' diseases of the Skin: clinical Dermatology. Saunders Elsevier. 2006. 0-7216-2921-0.

2. Mundinger GS. Halo Phenomenon. New Eng J Med. 2014;3;2622.

3. Nordlund JJ. Vitiligo: a review of some facts lesser known about depigmentation. Indian J Dermatol. 2011;56:180-9.

4. Ortonne JP, Nordlund J. Mechanisms that cause abnormal skin color. In: Nordlund JJ, Boissey RE, Hearing VJ, King RA, Oetting WS, Ortonne JP, editors. The Pigmentary System: Physiology and
Pathophysiology. Oxford: Blackwell Scientific Publishing; 2006. pp. 521-38.

5. Nordlund JJ, Ortonne J. The normal color of human skin. In: Nordlund JJ, Boissey RE, Hearing VJ, King RA, Oetting WS, Ortonne JP, editors. The Pigmentary System: Physiology and Pathophysiology. Oxford: Blackwell Scientific; 2006. pp. 504-20.

6. Byrne KT, Turk MJ. New Perspectives on the role of vitiligo in immune responses to melanoma. Oncotarget. 2011;2:684-94.

7. Bello-Quintero CE, Gonzalez ME, Alvarez-Connelly E. Halo nevi in Turner syndrome. Pediatr Dermatol. 2010;27:368-9.

8. What are the symptoms of Turner syndrome? Eunice Kennedy Shriver National Institute of Child Health and Human Development. 30 November 2012.

9. Sybert VP, McCauley E, McCauley. Turner's syndrome. N Engl J Med. 2004;351:1227-38.

10. Tjioe M, Gerritsen MJ, Juhlin L, van de Kerkhof PC. Treatment of vitiligo vulgaris with narrow band UVB $(311 \mathrm{~nm})$ for one year and the effect of addition of folic acid and vitamin B12. Acta Derm Venereol. 2002;82:369-72.

11. Teulings HE, Overkamp M, Ceylan E, Nieuweboer-Krobotova L, Bos JD, Nijsten T, et al. Decreased risk of melanoma and nonmelanoma skin cancer in patients with vitiligo: a survey among 1307 patients and their partners. Br J Dermatol.2013;168:162-71.

12. Gorton HL, Vogelmann TC. Ultraviolet radiation and the snow alga Chlamydomonas nivalis (Bauer) Wille. Photochem Photobiol. 2003;77:608-15.

13. Całkosiński I, Dobrzyński M, Rosińczuk J, Dudek K, Chrószcz A, Fita $\mathrm{K}$, et al. The use of infrared thermography as a rapid, quantitative, and noninvasive method for evaluation of inflammation response in different anatomical regions of rats. Biomed Res Int. 2015;2015:972535.

Copyright by Lorenzo Martini. This is an open access article distributed under the terms of the Creative Commons Attribution License, which permits unrestricted use, distribution, and reproduction in any medium, provided the original author and source are credited.

Source of Support: Nil, Conflict of Interest: None declared. 